\title{
Bäume im Palmengarten mit auffälligen Stämmen
}

\author{
Hilke Steinecke
}

Der Palmengarten verfügt über eine umfangreiche Gehölzsammlung. Im Freiland gedeihen zahlreiche einheimische sowie exotische Gehölze, darunter auch einige alte und große Bäume. Ein charakteristisches Merkmal von Bäumen ist ihr Stamm mit einer oft kräftigen Borke, die je nach Art z. B. glatt oder runzelig strukturiert oder z.B. aber auch rötlich, grünlich, grau, braun oder weiß gefärbt ist. Die nachfolgend kurz vorgestellten Bäume sollen diese Farbenvielfalt der Stämme verdeutlichen.

\section{Arbutus menziesii (Amerikanischer Erdbeer- baum, Ericaceae) hat ein weites Verbreitungs-} gebiet entlang der Westküste Nordamerikas von Kanada bis nach Kalifornien. Der bis $30 \mathrm{~m}$ hohe Baum wurde zu Ehren des schottischen Gärtners und Pflanzensammlers Archibald Menzies (1754-1842) benannt. Ihm wurde auch die ebenfalls im Westen Nordamerikas heimische Douglasie (Pseudotsuga menziesii) gewidmet. Die Blätter des immergrünen Gehölzes sind oberseits glänzend grün, unterseits blassgrün. Sie haben eine Lebensdauer von zwei Jahren und färben sich vor dem Abfallen leuchtend rot. Besonders auffällig ist die rotbraune Borke, die sich alliährlich schuppenartig vom Stamm ablöst und die darunterliegenden grünen Schichten freigibt. Erdbeerbäume gehen an ihren Wurzeln mit vielen Pilzen eine Mykorrhiza-Symbiose ein. Aus den kleinen weißen glockenförmigen Blüten gehen kleine, rot-orange Beerenfrüchte hervor. Diese werden von diversen Tieren gefressen. Der Erdbeerbaum befindet sich hinter dem Oktogon-Haus 1 in der Nähe des Betriebseingangs Miquelallee.

Acer griseum (Zimt-Ahorn, Sapindaceae) ist in Zentralchina endemisch, wo er in Mischwäldern wächst. Er wurde erst 1901 von ERNEST

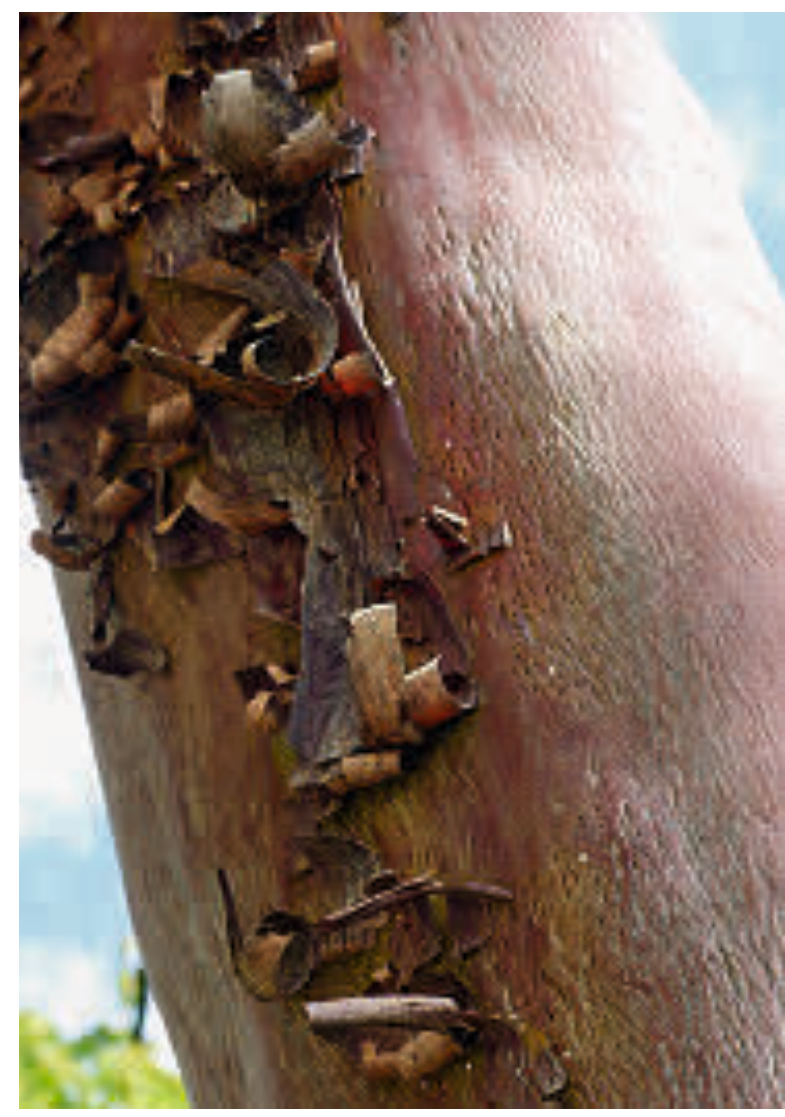

Wilson nach Europa eingeführt. Mit seinen dreizähligen Blättern, die sich im Herbst leuchtend rot färben, ist der Zimt-Ahorn nicht sofort als typischer Vertreter der Gattung Acer zu erkennen. Es handelt sich um einen kleinen, langsam wachsenden Baum bis 20 m Höhe, die er als Zierbaum in Mitteleuropa aber nie erreicht. Auffälligstes Merkmal ist seine zimtfarbene Borke, die sich besonders am Stamm und an älteren Seitenästen papierartig in langen Streifen abrollt. Die Blüten sind gelblich. Der Zimt-Ahorn ist zweihäusig, d. h. es gibt männliche und weibliche Individuen. Unser Baum setzt Früchte an, die gut $3 \mathrm{~cm}$ lange Flügel tragen und vom Wind ausgebreitet werden. Da eine männliche Pflanze fehlt, kommt es zu keiner Bestäubung und die Samen bleiben taub. Der Zimt-Ahorn wächst am Quellweiher in der Nähe des Hauses Leonhardsbrunn. 

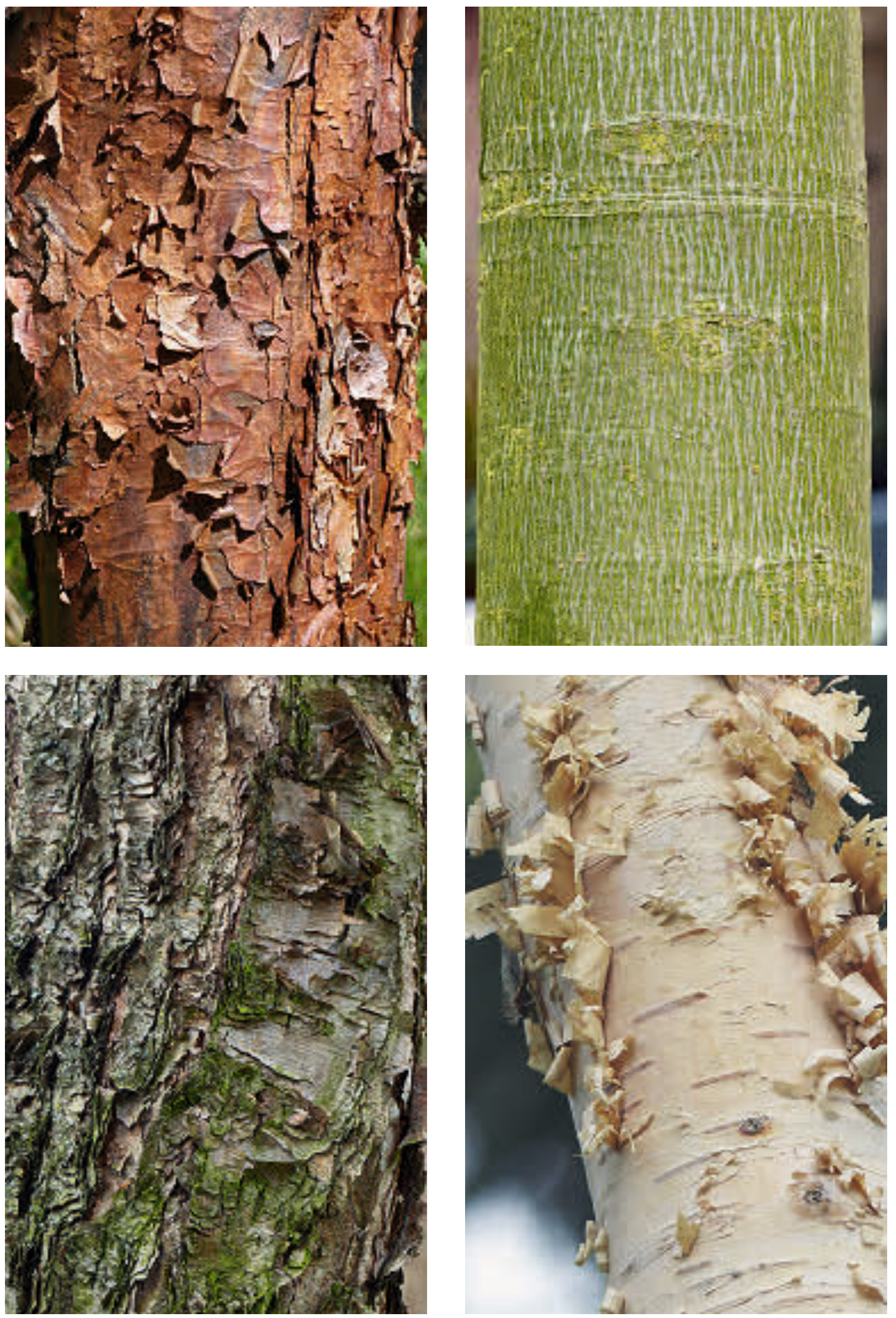
Firmiana simplex (Sonnenschirmbaum, Malvaceae) ist eine von 16 Arten der Gattung Firmiana, die fast alle in Asien heimisch sind. Mit dem Gattungsnamen ehrte Carl voN Linné den Österreicher KarL JOSEPH vON Firmian (1716-1782), Generalgouverneur der Lombardei. Der bis $20 \mathrm{~m}$ hohe, sommergrüne Baum stammt vermutlich nur aus China und wurde schon früh in Japan eingeführt. Über Japan kam Firmiana im 18. Jh. erstmals nach Europa (England). Auffällig ist der selbst im Alter glatte Stamm, der bei jüngeren Exemplaren grün gefärbt ist. Die großen, meist fünflappigen Blätter falten sich bei Hitze und Trockenheit entlang der Hauptadern schirmartig zusammen, daher der Name Sonnenschirmbaum. Die kleinen gelblichgrünen Blüten sind einzeln wenig spektakulär, wirken in ihrer Masse aber sehr attraktiv. Sie werden im Frühsommer in endständigen, bis rund $40 \mathrm{~cm}$ langen, überhängenden Rispen gebildet, die den Baum wie ein Schleier überziehen können. Aus den Blüten gehen fünf freie hellbraune Bälge hervor, die sich bereits vor der Reife öffnen und an ihren Rändern wenige erbsengroße Samen tragen, die bei einigen Firmiana-Arten zur Herstellung eines koffeinhaltigen Tees genutzt werden. Firmiana simplex wächst schnell und ist robust; ein Exemplar, das durch den Atombombenangriff von Hiroshima 1945 stark geschädigt wurde, ist ein Jahr später wieder ausgetrieben. Der tropisch anmutende Baum wird in Deutschland nur selten gepflanzt, weil er oft für nicht winterhart gehalten wird. Unsere beiden Exemplare haben bereits extrem kalte Winter ohne Winterschutz überlebt, im Jahr 2015 hat der Baum in der Nähe des Wasserbeckens vor dem Direktionsgebäude erstmals geblüht. Firmiana simplex steht außer am Wasserbecken auch seitlich des Eingangsschauhauses gegenüber dem Tropicarium.

Abb. 2 (Seite 68 oben links): Acer griseum.

Abb. 3 (Seite 68 oben rechts): Firmiana simplex.

Abb. 4 (Seite 68 unten links): Betula nigra.

Abb. 5 (Seite 68 unten rechts): Betula papyrifera.
Betula nigra (Schwarz-Birke, Betulaceae) ist ein meist mehrstämmiger Baum bis 25 m Höhe. Im Gegensatz zu den weißen Stämmen der meisten Birken-Arten hat Betula nigra dunkel rotbraune Stämme. Die Borke löst sich vom Stamm und ist dann kraus aufgerollt. In ihrer Heimat im Osten Nordamerikas besiedelt sie Flussauen und temporär überflutete Standorte (daher auch der englische Name river birch). Die Schwarz-Birke ist ein Pioniergehölz. Ihre kleinen geflügelten Früchte lösen sich im Sommer aus den Fruchtkätzchen und werden durch den Wind ausgebreitet. Sie keimen schnell und oft in Massen dort, wo das Land nicht mehr überflutet ist. Wegen ihrer attraktiven Borke und der gelben Herbstfärbung wird die bei uns völlig frostharte Schwarz-Birke gelegentlich als Zierbaum gepflanzt. Das Holz wird für Rundhölzer, Furniere oder Parkett genutzt. Indianer nutzten den eingedickten Baumsaft ähnlich wie Ahornsirup als Süßstoff. Die innere Rinde diente in Notzeiten als Nahrung. Die Schwarz-Birke steht zwischen Liegewiese und Steingarten.

Betula papyrifera (Papier- oder Amerikanische Weiß-Birke, Betulaceae) stammt aus dem Norden Nordamerikas und ist Landesbaum der kanadischen Provinz Saskatchewan und des amerikanischen Bundesstaates New Hampshire. Sie wird in ihrer Heimat $20 \mathrm{~m}$ hoch bei einem Stammdurchmesser von rund $80 \mathrm{~cm}$. Wie auch andere Birken-Arten entwickelt sie vor dem Laubaustrieb kätzchenförmige Blütenstände und wird durch den Wind bestäubt. Da sie bei uns völlig frosthart ist, wird sie hier auch als Ziergehölz gepflanzt. Auffällig ist ihre attraktive leuchtend weiße Borke, die sich in horizontalen Streifen vom Stamm löst (Ringelborke). Die weiße Farbe ist auf den Inhaltsstoff Betulin zurückzuführen. Sogenannte Birkenrinde ist wasserundurchlässig und gleichzeitig elastisch, weshalb sie ein wertvoller Rohstoff ist. Amerikanische Ureinwohner nutzten Birkenrinde wie Papier zum Schreiben. Außerdem wurde sie zum Abdecken von Dächern sowie zum Bau von Kanus verwendet. Die Papier-Birke findet sich im Gehölzstreifen zwischen Liegewiese und Bootsweiher. 

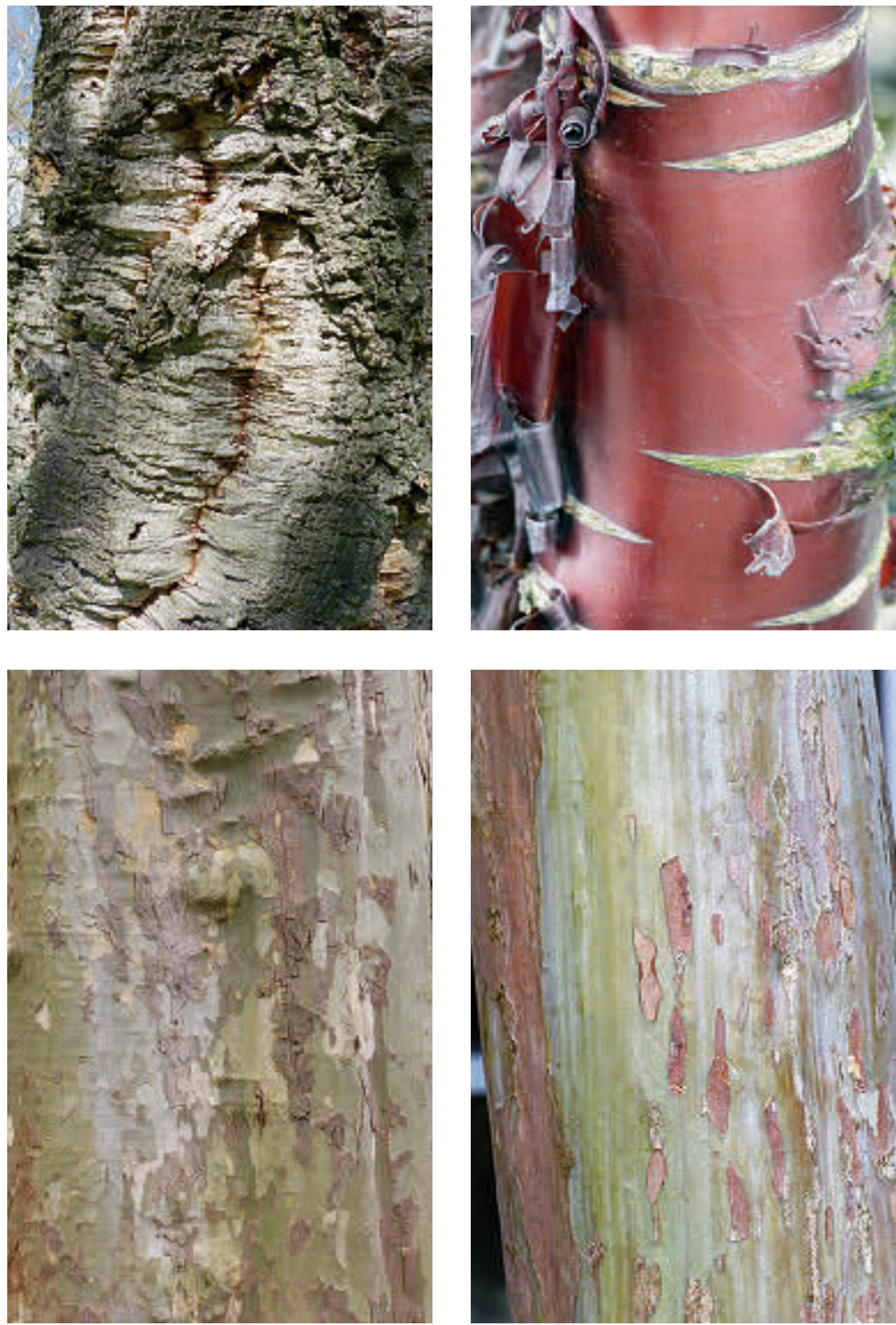
Quercus suber (Kork-Eiche, Fagaceae) ist in SW-Europa und NW-Afrika heimisch. Die immergrüne Kork-Eiche kann bis 20 m hoch werden. Kork-Eichen werden erstmals nach zwanzig Jahren geschält, danach alle neun Jahre. Kork ist vielseitig verwendbar: als Flaschenkork, Fußbodenkork, Tapeten oder Granulat z. B. für die Produktion von Sohlen und Fußbetten. In Portugal werden traditionell auch Handtaschen und Portemonnaies aus Kork hergestellt. Die Borke wird bis $15 \mathrm{~cm}$ dick. Geschält werden kann die Eiche vom 20. bis zum 150. Lebensjahr. Ihr Stamm zeigt nach dem Schälvorgang eine rötlichbraune Färbung. Der Kork ist eine Anpassung an den Standort. Er schützt die Eiche vor Feuer. Das Holz ähnelt bezüglich Härte, Dichte und Schwere dem Holz von Quercus ilex (Stein-Eiche) und Quercus coccifera (Kermes-Eiche). Es ist aber noch deutlich ringporiger. Es wird in der Tischlerei, zum Wagenbau, als Brennholz und zum Schiffsbau verwendet. Kork-Eichen-Wälder sind wertvolle, artenreiche Lebensräume. Durch Ersatz der Flaschenkorken durch andere Materialen verschwinden Kork-Eichen zunehmend aus der Kulturlandschaft. Die KorkEiche gedeiht am Seitenweg zwischen Tropicarium und Eingangsschauhaus.

Platanus $\times$ bybrida (Gewöhnliche Platane, Platanaceae) ist eine in der Mitte des 17. Jh. entstandene Kreuzung zwischen der Morgenländischen ( $P$. orientalis) und der Amerikanischen ( $P$. occidentalis) Platane, wobei ihr Ursprung unklar ist. Sie wird $45 \mathrm{~m}$ hoch und 300 Jahre alt. Sie ist robust und wird häufig als Straßenbaum sowie in Parks gepflanzt. Ihre gelappten Blätter erinnern an Blätter des Ahorns; sie sind aber lederiger und stehen wechselständig,

Abb. 6 (Seite 70 oben links): Quercus suber.

Abb. 7 (Seite 70 oben rechts): Platanus $\times$ hybrida

Abb. 8 (Seite 70 unten links): Prunus serrula.

Abb. 9 (Seite 70 unten rechts): Lagerstroemia indica während Ahorne gegenständig beblättert sind. Etwa mit dem Laubaustrieb erscheinen die Blütenstände. Aus den weiblichen, kugeligen Blütenständen gehen etwa $3 \mathrm{~cm}$ breite kugelige Sammelfruchtstände hervor. Platanen haben eine Schuppenborke. Dabei blättert die Borke in dünnen Platten ab. Dadurch entsteht auf dem Stamm ein buntes Muster aus weißlichen, grünen und grauen Feldern. Die größte Platane des Gartens steht am Palmenhaus in der Nähe des Eingangs in die Ausstellungsgalerien.

Prunus serrula (Mahagoni-Kirsche, Rosaceae) stammt aus China. Sie wächst strauchoder baumförmig und erreicht am natürlichen Standort eine Höhe von $12 \mathrm{~m}$. Im Frühling wird sie wegen ihrer weißen Blüten als Zierpflanze sehr geschätzt. In Parks und Gärten wird sie vor allem aber auch wegen ihrer glänzenden Borke (Spiegelborke), die während des ganzen Jahres attraktiv ist, gepflanzt. Sie ist glatt, mahagonibraun und durch Lentizellen hell genarbt. Die Wildart bildet eirunde Früchte, die 0,6 cm lang und leuchtend rot gefärbt sind. Mahagoni-Kirschen wachsen in der Palmengartenstraße auf dem Weg zum Blumenparterre.

\section{Lagerstroemia indica (Kreppmyrte, Lythraceae)}

Die Kreppmyrte ist ein kleiner Baum bis $10 \mathrm{~m}$ Höhe. Seine Heimat ist China. Im Mittelmeergebiet wird er wegen seiner attraktiven rosa Blüten häufig als Zierbaum gepflanzt. Da er nur bedingt winterhart ist, kann er in Deutschland nur in den mildesten Gebieten ohne Winterschutz draußen überleben. Bei Spätfrösten erfrieren häufig die bereits im Vorjahr angelegten Blütenknospen. Die gekräuselten, duftlosen Blüten erinnern etwas an Krepp-Papier, was sich auf den deutschen Namen bezieht. Auffällig ist bei älteren Exemplaren die rötliche, abblätternde Borke. Sie ist sehr glatt, daher auch die Bezeichnung Affenrutschbaum. Ein Verwandter der Kreppmyrte, der Blutweiderich, ist eine bei uns heimische Staude an Ufern von Bächen und Seen. Kreppmyrten stehen am Eingang zum Tropicarium (Weltkugel). 